\title{
Experimental Study on Gear Extrusion Machining Process of Gearbox Synchronizer Gear Hub
}

\author{
SHI Yaochen 1, a , LI Zhanguo ${ }^{2, b}$, LIN Dan ${ }^{3, c}$, LI Qinghua ${ }^{1, d}$ \\ ${ }^{1}$ Changchun University, Changchun, 130022, China \\ ${ }^{2}$ Changchun University of technology, Changchun, 130022, China; \\ ${ }^{3}$ Changchun University of Science and Technology, Changchun, 130022, China \\ a250793721@qq.com, b40881220@qq.com, ${ }^{c}$ 809981371@qq.com, \\ d18237930@qq.com
}

\begin{abstract}
This study treats the synchronizer gear hub gear extrusion machining process for J6L medium truck. The CA6140 lathe has been reformed into a gear extrusion machine and the experimental research about the gear extrusion plastic forming processing has been did. The tooth surface roughness and hardness after the gear extruding process under the given process parameters had also been analyzed. The experimental results showed that the surface roughness had been improved after the gear extruding processing, and the tooth surface hardness was became higher compare with the original tooth surface. It is obviously that the gear extrusion machining process can improve the tooth surface quality of the gear hub and get a better process result.
\end{abstract}

KEYWORDS: Synchronizer gear hub; Gear extrusion machining process; The tooth surface roughness and hardness

\section{INTRODUCTION}

As an important part of gearbox, the role of a synchronizer is making two gears meshing with each other at the same speed through the friction contact of friction pairs, and reducing the impact of gears in the shifting process in order to prolong the service life of the gears and make the shift operation smoothly[1,2]. In the synchronizer, the external spline of the gear hub and the internal spline of the gear sleeve are always connecting with each other, and relatively slip along the axis when shifting. So the property of the gear hub will directly affects the shift performance [3].

In order to improve the stability and the carrying capacity of gear hob in the transmission process the gear extrusion machining process is proposed. It not only can improve the tooth surface quality of the gear hob effectively, but also can achieve the requirement of thinning the tooth surface [4]. The gear extrusion 
machining process, also called the finishing process of gear. Gear hub and extrusion wheel are respectively installed on two parallel rotary spindles. At the beginning stage, applied radial gear extrusion force $\mathrm{F}$ to the extrusion wheel in order to let the extrusion wheel gradually get close to the workpiece and engage with it. At the forming stage, the gear hub rotates actively. The extrusion wheel acts as driven wheel and keeps the biggest gear extrusion force constantly (keeping the constant compression unchanged). The tooth surface of the gear hub produces plastic deformation under the radial extrusion force, and finally gets the involute tooth profile. At the finishing stage, keep the extrusion wheel at its biggest feed stroke unchanged and let the gear hub continue rotating to complete the final modification of gear hub surface, as shown in Fig.1. Gear extrusion method is a kind of near-net shaping technology[5]. Its advantages are high productivity, low cost, no chip and the machined surface roughness is low[6]. At present, a series of experiments and researches about gear extrusion machining process had been carried out both here and abroad. In 2006, Chu Yan has proved that by using the gear extrusion method, the variation of the gear normal line can keep between $0.2 \sim 0.5 \mathrm{~mm}$, and the radial pulsation of the gear ring is between 0.01 0.03mm[7]. The SCHLER company of Germany has proved that the gear precision can reach $6 \sim 7$ level by using the gear extrusion method, the tooth thickness error is only about $\pm 0.05 \mathrm{~mm}$ and the production efficiency can reach 25 30 piece in one minute[8].

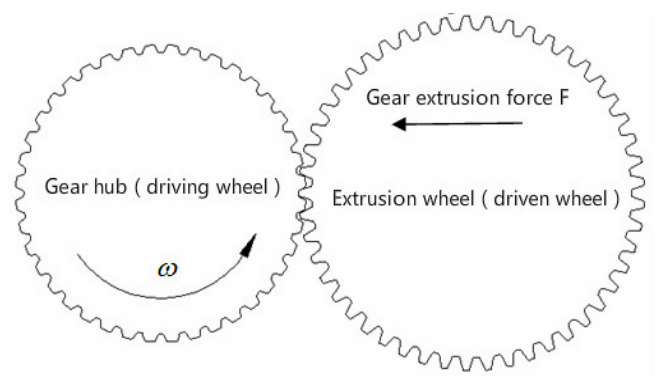

Figure 1 Working principle diagram of gear extruding processing

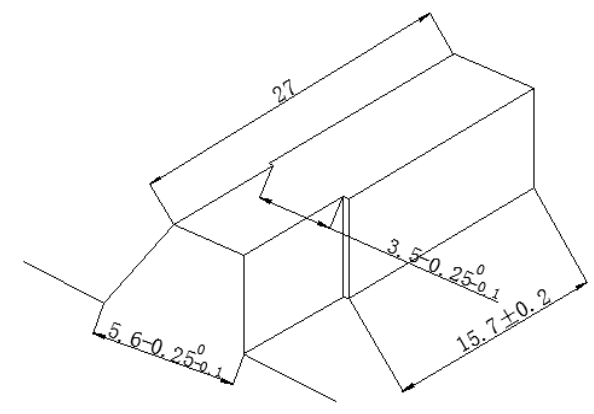

Figure 2 The machining dimension parameters of the gear hub

\section{Published by Francis Academic Press, UK}


This study treats the synchronizer gear hub gear extrusion machining process for J6L medium truck. Reduced the gear tooth thickness of $27 \mathrm{~mm}$ with the width of $0.25 \sim 0.35 \mathrm{~mm}$, and the original length of the tooth width retained $15.7 \pm 0.2 \mathrm{~mm}$ in the direction of the tooth width. Fig. 2 shows the machining dimension parameters.

\section{PARAMETERS AND EXPERIMENTAL CONDITIONS OF GEAR HUB GEAR EXTRUSION PROCESSING}

In order to analysis the influence of gear extrusion process on the hardness and precision of the gear hub surface, an experimental research has been done on a lathe in this study. The parameters of the gear hub are as follow: the number of teeth $\mathrm{z}$ $=40$, the modulus $m=2.5$, the pressure angle of pitch circle $\alpha=20^{\circ}$, the tip diameter $d_{z}=103.5 \mathrm{~mm}$, the root diameter $d_{f}=99 \mathrm{~mm}$. The parameters of the extrusion wheel are as follow: the number of teeth $\mathrm{z}=49$, the modulus $m=2.5$, the pressure angle of pitch circle $\alpha=20^{\circ}$, the tip diameter $d_{z}=123.46 \mathrm{~mm}$, the root diameter $d_{f}=115.2 \mathrm{~mm}$. The Workpiece has been treated through quenching and tempering treatment. The gear hub had been machined under a speed of $280 \mathrm{r} / \mathrm{min}$ and the gear extrusion force of 70KN. Fig. 3 shows the gear hub after turning 10 circles.

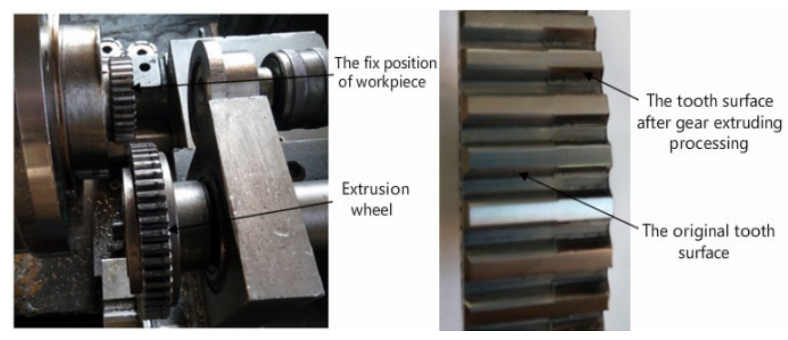

Figure 3. The tooth surface of gear hub after gear extruding processing

\section{THE EXPERIMENT RESULT ANALYSIS OF GEATR EXTRUSION MACHINING PROCESS}

\subsection{Roughness analysis of the synchronizer gear hub tooth surface}

In order to analysis the influence of gear extrusion processing on tooth surface hardness that 8 workpieces had been machined through gear extrusion process. Choose 3 points from the gear top, the tooth side and the tooth root of each tooth profile respectively to measure their surface roughness before and after the gear extrusion and took their average value as the surface roughness value of the workpiece. Fig.4 shows the measuring point. 


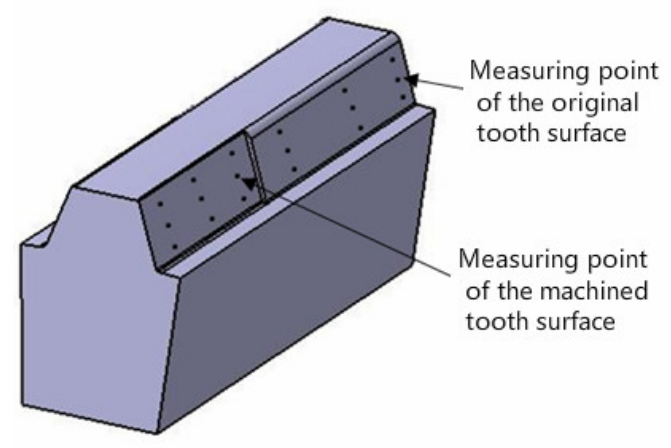

Figure 4. Roughness measuring point of the gear tooth surface

In the fig. 5 shows the roughness curve of gear tooth surface before and after the gear extruding process.

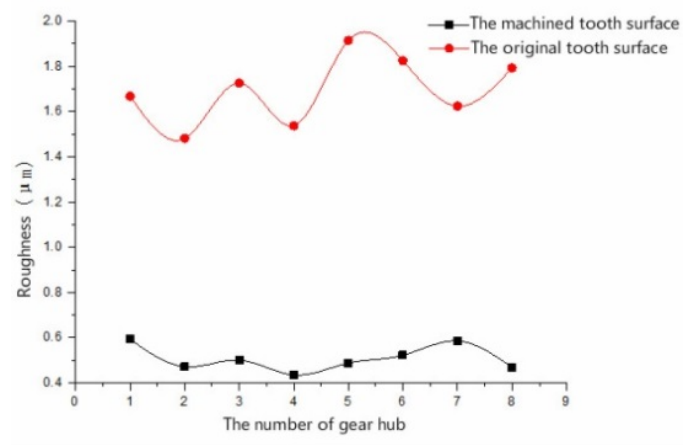

Figure 5. The roughness value curve of the gear hub tooth surface

The maximum value of the original tooth surface roughness is $1.9135 \mu \mathrm{m}$, and the minimum value is $1.4816 \mu \mathrm{m}$. The maximum value of the tooth surface roughness after the gear extruding process is $0.5930 \mu \mathrm{m}$, and the minimum value is $0.4359 \mu \mathrm{m}$. The surface roughness of the machined gear hub is better than the original one. The cause of this phenomenon is that the plastic deformation of the gear surface leaded to the cold hardening and generated residual compressive stress in the tooth surface. After plastic forming of the gear hub, the internal metal tissue of the gear hub had not been cut off and the repeated extrusion of the extrusion wheel eliminated the slight uneven of the tooth surface. So the surface roughness being improved after gear extruding processing. 


\subsection{Hardness analysis of the synchronizer gear hub tooth surface}

Chose a complete tooth of a gear hub was being machined, and measured its Vickers hardness through micro hardness tester by applying the load of 500g on its cross section. Choose a number of measuring points from the cross section in order to analysis the cold hardening phenomena of the gear tooth after gear extruding process precisely. Fig.6. shows the measuring point. And the measured equal hardness curve is expressed on the cross section of the tooth profile, as shown in Fig.7.

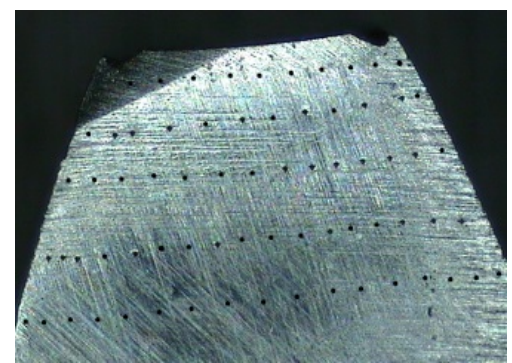

(a) Measuring point of cross section

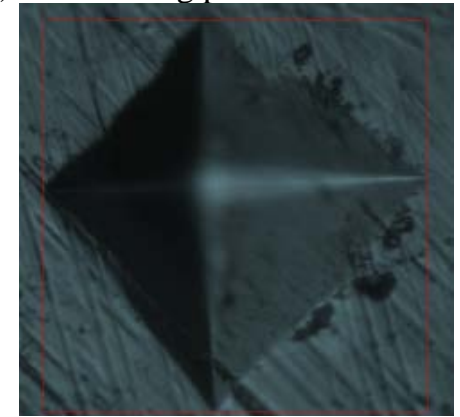

(b) The enlarged drawing of the measuring point

Figure6. Cross section micro hardness measurement point of gear hub

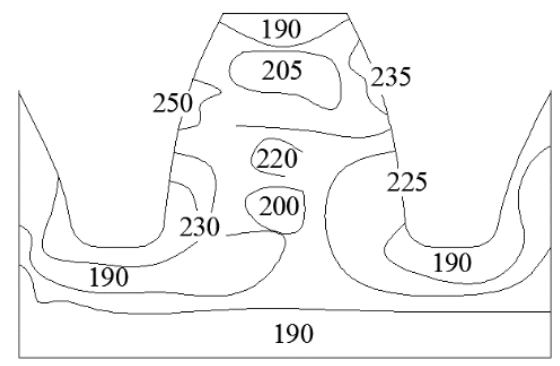

Figure 7. Hardness distribution of cross section of the gear bub tooth profile

\section{Published by Francis Academic Press, UK}


(a) In order to analysis the cold hardening effect of gear hub tooth surface, choose 10 points of the gear top, the tooth side and the tooth root separately from the measured date and calculated the average valve. Fig. 8 shows the result after the curve fitting.

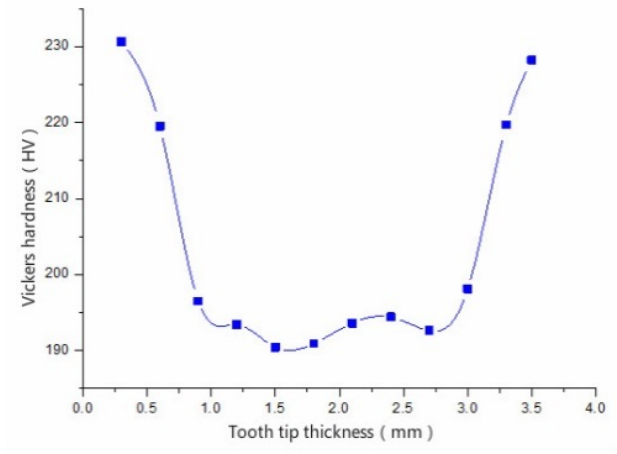

(b) Distribution of tooth tip hardness along the tooth thickness

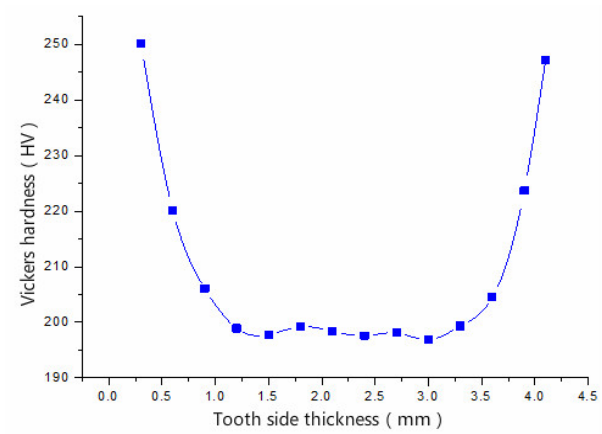

(b) Distribution of tooth side hardness along the tooth thickness

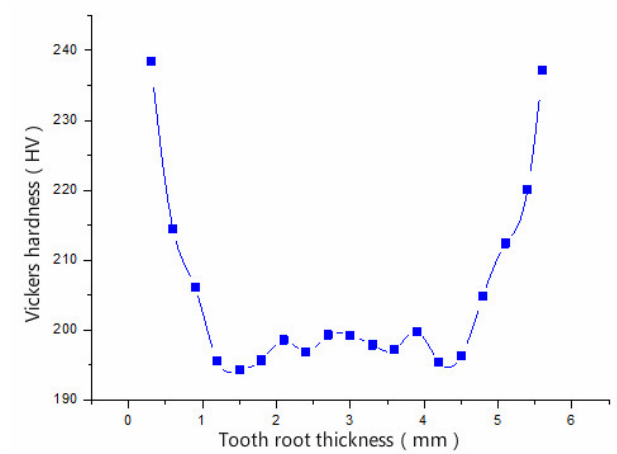

(c) Distribution of tooth root hardness along the tooth thickness

Figure8. Distribution of tooth surface hardness along the tooth thickness 
Fig.7 and Fig.8 shows that the hardness distribution of the gear hub was gradually decreased from the surface to the inside, and the distribution laws of every parts of the gear hub tooth profile were almost the same. The hardness of the gear top, the tooth side and the tooth root could reach HV230, HV250, HV240. During the gear extruding process, the surface material generated violent plastic deformation. The deformation leaded to the cold hardening phenomenon and the increase of the tooth surface hardness. The depth of the hardened layer was about $0.6 \sim 0.9 \mathrm{~mm}$.

\section{CONCLUSIONS}

This study treats the synchronizer gear hub gear extruding processing technology of J6L medium truck. The gear compression test was carried out with the modified CA6140 lathe. The experiment proved the influence of gear extruding process on the gear hub tooth surface roughness and hardness:

(1) In this study, the experiment had been done to analysis the influence law of surface roughness after the gear extruding processing. The maximum value of the original tooth surface roughness is $1.9135 \mu \mathrm{m}$, and the minimum value is $1.4816 \mu \mathrm{m}$. The maximum value of the tooth surface roughness after gear extrusion is $0.5930 \mu \mathrm{m}$, and the minimum value is $0.4359 \mu \mathrm{m}$. The result proved that the surface roughness being improved after the gear extruding processing.

(2) In this study, the experiment had been done to analysis the influence law of tooth surface hardness after the gear extruding processing. The original tooth surface hardness is HV190. Because of the cold hardening phenomena, the hardness value arrives HV250 after the gear extruding process and the depth of the hardened layer is about $0.6 \sim 0.9 \mathrm{~mm}$.

\section{REFERENCES}

[1] Xu Wanli, Zhao Wei, Zhang Xueming, Xu Ximeng, Li Bin. Analysis on failure process and failure Mechanism of transmission synchronizer [J]. Journal of Mechanical Engineering, 2009(12):189 192

[2] Xie Zongyu. Auto transmission synchronizer test rig developed[J]. Equipment Manufacturing Technology, 2015(3):58 60

[3] Xu Liyou, Cao Qingmei, Zhou Zhili, Niu Yi. Review on the Development of tractor Transmission[J]. Journal of Agricultural Mechanization Research, 2009(12):189 192

[4]Zhang Hua, Xu Linlin, Han Yinan, Yang Jianjun and Ma Jiangmin. Design and machining experiment of constant-depth tooth spiral bevel gear [J]. Journal of Mechanical Transmission, 2014, 8, 38(8):157 159

[5] Yin Xinying. A kind of high efficiency gear finish machining technology-gear extruding process[J]. Journal of Hebei Institute of Mechano-Electric Engineering. 1998,15(2):81 85 
Academic Journal of Engineering and Technology Science ISSN 2616-5767 Vol. 1, Issue 1: 1-8, DOI: 10.25236/AJETS.020001

[6Li Yanbin, Zheng Peng. Precise ausform methods used for machining gears with hard surface $[\mathrm{J}]$. Journal of Shenyang university of technology, 2002, 12, 24(6):457 460

[7]Chu Yan, Huang Suzhen. Design of a new type gear cold extruding machine[J].New technology and product, 2006(1):84 85

[8]Zhang Lihui. Study on warm precision forging of spur gears with big modulus and high boss [M]. Hefei Polytechnic University.2005. 